\title{
邻炔基苯胺的电化学环化合成 3-碘吲哚
}

\author{
徐鹤华 $a$ 孟祥太 $a$ 郑 煜 ${ }^{a}$ 罗金岳*,a 黄申林*,a,b \\ ( ${ }^{a}$ 南京林业大学化学工程学院 江苏省林业资源高效加工利用协同创新中心 南京 210037) \\ ( ${ }^{b}$ 林产化学与材料国际创新高地 南京 210037)
}

\begin{abstract}
摘要 吲哚及其衍生物是一类重要的杂环化合物，也是多种天然产物和合成药物的骨架分子. 3-硒吲哚在其选择性功 能化中充当着重要的中间体. 电化学氧化廉价易得的碘盐, 促使邻炔基苯胺分子内环化, 便捷地合成了一系列 3-磑吲 哚类化合物, 目标化合物可获得良好至优秀的产率. 该方法条件温和, 官能团适用范围广, 无需额外添加氧化剂和碱, 是一条操作简便、绿色可持续地合成 3-碘吲哚类化合物的途径.
\end{abstract}

关键词＼cjkstart电化学合成; 环化反应; 3-碘吲哚; 邻炔基苯胺

\section{Electrochemical Annulations of o-Alkynylanilines for Synthesis of 3-lodoindoles}

\author{
$\mathrm{Xu}$, Hehua $^{a} \quad$ Meng, Xiangtai $^{a} \quad$ Zheng, Yu $^{a} \quad$ Luo, Jinyue $^{*, a}$ Huang, Shenlin ${ }^{*, a, b}$ \\ ( ${ }^{a}$ Jiangsu Co-Innovation Center of Efficient Processing and Utilization of Forest Resources, \\ College of Chemical Engineering, Nanjing Forestry University, Nanjing 210037) \\ ( ${ }^{b}$ International Innovation Center for Forest Chemicals and Materials, Nanjing 210037)
}

\begin{abstract}
Indole, as one of the most important nitrogen-containing heterocyclic compound, is found widely in various natural products and synthetic drugs. 3-Iodoindoles play an important role as intermediate in the selective functionalization of indoles. 3 -iodoindoles could be conveniently synthesized by electrochemical annulations of $o$-alkynylanilines with readily available iodide salt. This sustainable electrolysis strategy provides an efficient access to a series of 3-iodoindoles in good to excellent yields under external oxidant-free, base-free conditions and features broad functional group tolerance.

Keywords electrosynthesis; annulation; 3-iodoindoles; $o$-alkynylanilines
\end{abstract}

吲哚及其衍生物是一类重要的杂环化合物, 广泛存 在于天然产物和药物分子中 ${ }^{[1]}$. 吲哚卤化物是实现吲哚 功能化的重要中间体. 其中, 利用过渡金属催化的交叉 偶联实现碳-卤键的选择性功能化 ${ }^{[2]}$, 从而构建一系列 含氟 $[2 b]$ 、嶰 $[2 \mathrm{c}]$ 、吡喃环 ${ }^{[2]}$ 等吲哚衍生物. 近年来, 将碘 引入吲哚或者直接构建 3-碘吲哚一直受到化学工作者 的广泛关注 ${ }^{[3]}$. 如 Scheme 1 所示, Barluenga 课题组 $\left.{ }^{[3]}\right]$ 在 2003 年报道了双(吡啶)四氟嗍化碘 $\left(\mathrm{IPy}_{2} \mathrm{BF}_{4}\right.$ ) 促进邻炔基 苯胺的分子内环化, 在低温 $\left(-60{ }^{\circ} \mathrm{C}\right)$ 下构建一系列 3碘吲哚类化合物. 随后 Knight 课题组 ${ }^{[3 b]}$ 建立了一个更 为简单的策略, 利用硒单质和碳酸钾在更为温和 $(0 \sim$ $\left.20{ }^{\circ} \mathrm{C}\right)$ 的环境下合成此类化合物. 2015 年, 邓军课题组 [3c]报道了通过三苯基膦催化的 $N$-碘代丁二酰亚胺(NIS)
促进合成 3-碘吲哚的方法. 尽管 3-碘吲哚的合成已经取 得了很大的进展, 但目前已有方法需要使用有毒和腐蚀 性的碘单质或过渡金属、三苯基膦等催化剂. 由此可见, 开发一种绿色替代方法合成 3-碘吲哚仍具有重要意义.

过去的几十年里, 因其具有良好的环境效益和经济 可行性, 电化学合成 ${ }^{[4]}$ 受到越来越多有机合成工作者的 关注, 并成功应用于多种重要的有机合成反应 ${ }^{[5]}$. 有机 电化学合成以电子作为 “试剂”，避免了有毒、有害氧 化剂或还原剂的使用 ${ }^{[6]}$. 电化学可以通过改变电流或电 压来调节反应的氧化还原能力, 更自由地控制反应的进 程. 近年来, 碘盐阳极氧化生成的碘自由基、碘单质、 碘正离子等代替有机高价碘或含碘催化剂与氧化剂组 合的策略，被应用在多种化合物的合成上 ${ }^{[7]}$. 最近，屠

* Corresponding authors. E-mail: luojinyue@njfu.com.cn; shuang@njfu.edu.cn Received December 8, 2021; revised December 19, 2021; published online December 22, 2021. Project supported by the National Natural Science Foundation of China (No. 32171724). 国家自然科学基金(No. 32171724)资助项目. 


\section{Previous work}<smiles>[R]C#Cc1cc[R]([R])cc1N[R]</smiles>
$\mathrm{IPy}_{2} \mathrm{BF}_{4} / \mathrm{I}_{2} / \mathrm{NIS}$

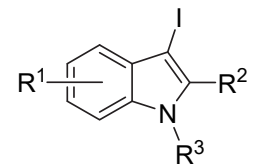

This work<smiles>[R]C#Cc1ccc([R])cc1N[Sb]</smiles>
$\frac{\text { TBAI, Electrolysis }}{\mathrm{MeCN} / \mathrm{H}_{2} \mathrm{O}, \text { r.t. }}$

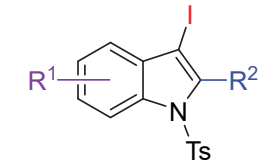

external oxidant-free, external base-free, mild conditions, 21 examples up to $98 \%$ yield

图式 1 通过碘基试剂合成 3-碘吲哚

Scheme 1 Synthesis of 3-iodoindoles by iodide-based reagents

树江和姜波课题组 ${ }^{[8]}$ 报道了一种以碘化铵为碘源、乙醇 作溶剂, 电化学合成 3-碘吲哚类化合物的方法. 他们主 要研究了一类结构独特的邻芳基取代炔基苯胺的环化 反应, 其中炔基连接的 2-羟基菜被认为是该电化学成环 的关键因素. 因此, 开发一种更具普适性的绿色替代方 案合成 3-碘吲哚类化合物仍是迫切的.

我们课题组前期实现了电化学条件下生成碘化物 介导炔烃和亚磺酸钠的反应, 从而构建了一系列炔基砜 类化合物 ${ }^{[9]}$. 在此基础上, 我们在电化学条件下, 以廉 价易得的 TBAI 为碘源, 以乙腈和水为混合溶剂, 开发 了经分子内环化的策略来合成 3-碘吲哚的新方法. 该方 法具有以下几个方面的优势: (1)无需额外的氧化剂、过 渡金属催化剂以及碱; (2)相较于先前的电化学合成方法 有更广泛的底物范围; (3)大部分目标产物可以在体系中 析出, 只需过滤就能得到高纯度的产物.

\section{1 结果与讨论}

\section{1 反应条件的优化}

首先, 以化合物 1a 为模型底物, 乙腈和水作为混合 溶剂, 通有 $3 \mathrm{~mA} / \mathrm{cm}^{2}$ 恒定电流的未分隔电解池内考察 反应条件. 如表 1 所示, 在 TBAI 作为电解质和碘源的 条件下, 反应能以 $65 \%$ 的液相产率顺利转化为预期的吲 哚产物 2a (Table 1, Entry 1). 但随着 TBAI 的消耗, 电压 逐渐升高, 原料无法全部转化. 为了保持反应体系电 流、电压的稳定, 添加高氯酸锂 $\left(\mathrm{LiClO}_{4}\right)$ 作为辅助电解 质，原料可以完全转化，以 $89 \%$ 的产率获得化合物 $\mathbf{2 a}$ (Table 1, Entry 2). 随后, 尝试其它电极在该反应中的表 现. 在使用石墨片电极作为阳极和镍片电极作为阴极的 条件下，以 68\%的产率获得了化合物 2a (Table 1, Entry 3). 当阳极和阴极都为石墨片电极时, 目标产物 2a 的产 率达到 82\% (Table 1, Entry 4). 相较于石墨片电极和镍 片电极, 当铂片为电极的阳极和阴极时产率最高. 在确
定了以铂片为阳极和阴极电极材料之后, 对辅助电解质 进行了篎选，实验发现大部分电解质在该反应中都是兼 容的. 当阴离子为六氟磷酸 $\left(\mathrm{PF}_{6}^{-}\right)$时都有着不错的表现 (详见辅助材料, Table S1). 其中, 使用六氟磷酸钾 $\left(\mathrm{KPF}_{6}\right)$ 的效果最好(Table 1, Entry 5), 能以 $94 \%$ 的产率得 到目标化合物 2a. 紧接着, 测试了不同碘盐在该反应中 的表现. 当使用碘化铵 $\left(\mathrm{NH}_{4} \mathrm{I}\right)$ 时反应效果较差, 仅得到 $17 \%$ 的目标产物(Table 1, Entry 6); 当使用碘化钾(KI)和 四甲基碘化铵 $\left(\mathrm{Me}_{4} \mathrm{NI}\right.$ )时产率分别为 73\%和 69\% (Table 1 , Entries 7,8). 当使用 1 equiv. TBAI 时，反应产率下降 至 67\% (Table 1, Entry 9). 增加 TBAI 用量至 2 equiv. 时 并没有获得更多的目标产物 2a (Table 1, Entry 10). 当体 系中不添加水反应无法顺利进行(Table 1, Entry 11). 不 通电的情况反应也无法向目标产物 2a 转化(Table 1, Entry 12). 综上所述, 最优的反应条件是：在室温下， 以铂片电极为阳极和阴极, 未分隔电解槽内添加 TBAI (1.5 equiv.), $\mathrm{KPF}_{6}$ (2.5 equiv.), $\mathrm{CH}_{3} \mathrm{CN}(5 \mathrm{~mL}), \mathrm{H}_{2} \mathrm{O}$ (1 $\mathrm{mL}$ ), 并使用 $3 \mathrm{~mA}$ 恒定电流电解.

表 1 反应条件优化 ${ }^{a}$

Table 1 Optimization of the reaction conditions

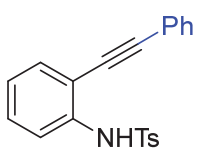

1a

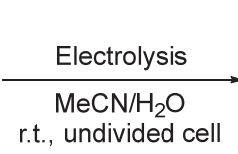

$2 a$

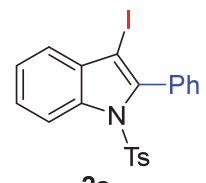

a

\begin{tabular}{ccccc}
\hline Entry & Electrode & Electrolyte & $\begin{array}{c}\text { Auxiliary } \\
\text { electrolyte }\end{array}$ & Yield $^{b} \%$ \\
\hline 1 & $(+) \mathrm{Pt} /(-) \mathrm{Pt}$ & $\mathrm{TBAI}$ & - & 65 \\
2 & $(+) \mathrm{Pt} /(-) \mathrm{Pt}$ & $\mathrm{TBAI}$ & $\mathrm{LiClO}_{4}$ & 89 \\
3 & $(+) \mathrm{C} /(-) \mathrm{Ni}$ & $\mathrm{TBAI}$ & $\mathrm{LiClO}_{4}$ & 68 \\
4 & $(+) \mathrm{C} /(-) \mathrm{C}$ & $\mathrm{TBAI}$ & $\mathrm{LiClO}_{4}$ & 82 \\
5 & $(+) \mathrm{Pt} /(-) \mathrm{Pt}$ & $\mathrm{TBAI}$ & $\mathrm{KPF}_{6}$ & 94 \\
6 & $(+) \mathrm{Pt} /(-) \mathrm{Pt}$ & $\mathrm{NH} 4 \mathrm{I}$ & $\mathrm{KPF}_{6}$ & 17 \\
7 & $(+) \mathrm{Pt} /(-) \mathrm{Pt}$ & $\mathrm{KI}$ & $\mathrm{KPF}_{6}$ & 73 \\
8 & $(+) \mathrm{Pt} /(-) \mathrm{Pt}$ & $\mathrm{Me} 4 \mathrm{NI}$ & $\mathrm{KPF}_{6}$ & 69 \\
9 & $(+) \mathrm{Pt} /(-) \mathrm{Pt}$ & $\mathrm{TBAI}^{c}$ & $\mathrm{KPF}_{6}$ & 67 \\
10 & $(+) \mathrm{Pt} /(-) \mathrm{Pt}$ & $\mathrm{TBAI}^{d}$ & $\mathrm{KPF}_{6}$ & 92 \\
$11^{e}$ & $(+) \mathrm{Pt} /(-) \mathrm{Pt}$ & $\mathrm{TBAI}^{\prime}$ & $\mathrm{KPF}_{6}$ & N.d. \\
$12^{g}$ & $(+) \mathrm{Pt} /(-) \mathrm{Pt}$ & $\mathrm{TBAI}^{f}$ & $\mathrm{KPF}_{6}$ & N.d. \\
\hline
\end{tabular}

${ }^{a}$ Standard conditions: constant current $=3 \mathrm{~mA}$, 1a $(0.3 \mathrm{mmol})$, TBAI $(1.5$ equiv), $\mathrm{KPF}_{6}$ (2.5 equiv.), $\mathrm{MeCN} / \mathrm{H}_{2} \mathrm{O}(6.0 \mathrm{~mL}, V: V=5 / 1)$ in an undivided cell with two platinum electrodes, r.t., $24 \mathrm{~h} .{ }^{b}$ Yields were determined by HPLC analysis with 1-nitronaphthalene as the internal standard. ${ }^{c}$ TBAI (1 equiv.). ${ }^{d}$ TBAI (2 equiv.). ${ }^{e}$ Without $\mathrm{H}_{2} \mathrm{O} .{ }^{f}$ N.d. $=$ not detected. ${ }^{g}$ Without current.

\section{2 反应底物的拓展}

在确定了最佳反应条件后，接着讨论了该反应的底 物适用性. 首先研究了邻炔基苯胺 $(\mathbf{1}) \mathrm{R}^{1}$ 位置的适用范 围. 如 Table 2 所示, 取代基的电子效应没有对反应产生 显著影响 $(2 \mathbf{b} \sim 2 \mathrm{i})$. 其中，供电子基团如甲基 $(\mathrm{Me})$ 、甲氧 
表 2 化合物 $\mathbf{2}$ 的底物范围 $a, b$

Table 2 Substrate scope for the synthesis of 2
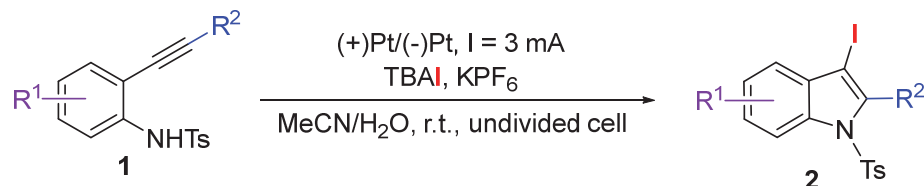

2 Ts

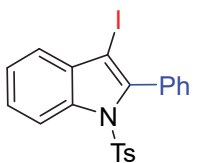

2a, $94 \%$<smiles>S[Y5]n1c(-c2ccccc2)c(I)c2cc(Br)ccc21</smiles>

2f, $84 \%$

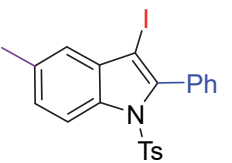

2b, $73 \%$

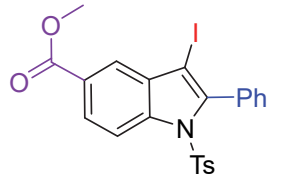

2g, $98 \%$

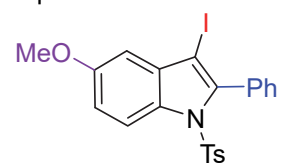

2c, $81 \%$<smiles>Fc1ccc2c(c1)c(I)c(-c1ccccc1)n2[AsH3-]</smiles>

2d, $74 \%$<smiles></smiles>

2e, $65 \%$

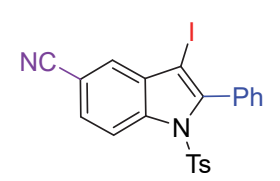

2h, $94 \%$

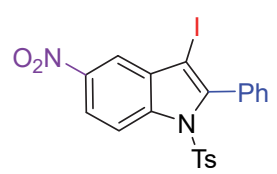

2i, $75 \%$

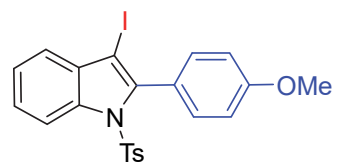

2j, 91\%<smiles></smiles>

2n, $84 \%$

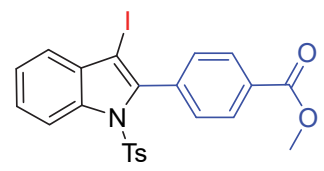

2r, $42 \%$

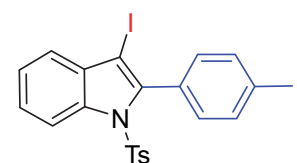

2k, $93 \%$<smiles></smiles>

2o, 95\%

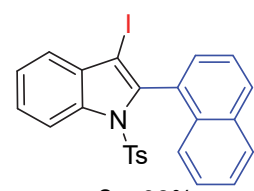

2s, $98 \%$<smiles>CC(C)(C)c1ccc(-c2c(I)c3ccccc3n2[AsH2])cc1</smiles>

2I, $92 \%$<smiles>Sc1c(-c2ccc(Br)cc2)n([AsH2])c2ccccc12</smiles>

2p, $81 \%$<smiles>Cc1c(I)c2ccccc2n1[AsH3-]</smiles>

2t, $87 \%$<smiles>Fc1ccc(-c2c(I)c3ccccc3n2[AsH2-])cc1</smiles>

2m, 89\%<smiles>N#Cc1ccc(-c2c(I)c3ccccc3n2[AsH2])cc1</smiles>

2q, $61 \%$

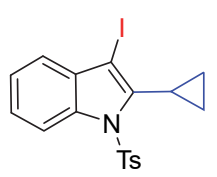

2u, $89 \%$

${ }^{a}$ Standard conditions: constant current $=3 \mathrm{~mA}, 1(0.3 \mathrm{mmol}), \mathrm{TBAI}(1.5$ equiv. $), \mathrm{KPF}_{6}(2.5$ equiv. $), \mathrm{MeCN} / \mathrm{H}_{2} \mathrm{O}(6.0 \mathrm{~mL}, V: V=5 / 1)$ in an undivided cell with two platinum electrodes, r.t., 24 h. ${ }^{b}$ Isolated yield.

基( $\mathrm{OMe})$ 以及弱吸电子基团 $(\mathrm{F}, \mathrm{Cl}, \mathrm{Br})$ 能以 $65 \% \sim 84 \%$ 的 产率转化为相应的 3-碘吲哚产物 $(\mathbf{2 b} \sim \mathbf{2 f})$. 值得一提的 是, 相较于先前的报道 ${ }^{[2 c, 3 c, 7]}, \mathrm{R}^{1}$ 是强吸电子基团时产率 不会有明显的下降. 例如, 酯基 $\left(\mathrm{CO}_{2} \mathrm{Me}\right)$ 和氧基 $(\mathrm{CN})$ 取 代的邻炔基苯胺分别以 98\%和 94\%的分离产率转化为 相应的 3-磑吲哚产物( $\mathbf{2 g}, \mathbf{2 h})$. 硝基 $\left(\mathrm{NO}_{2}\right)$ 取代的目标产 物(2i)可以以较好的产率(75\%)获得.

受这些结果的鼓舞, 进一步探究了 $\mathrm{R}^{2}$ 取代的邻炔 基苯胺的普适性(Table 2). 当 $\mathrm{R}^{2}$ 上含有供电子取代基 $(2 \mathbf{j} \sim 21)$ 时, 反应表现出了较好产率 $(91 \% \sim 93 \%)$. 而相 同位置为弱吸电子取代基 $(\mathrm{F}, \mathrm{Cl}, \mathrm{Br})$ 时，也能以 $81 \%$ $89 \%$ 的产率得到目标化合物(2 m, 2n, 2p). 间位取代的化 合物 (20)表现出更高的产率 (95\%). 连有强吸电子基团 $\left(\mathrm{CN}, \mathrm{CO}_{2} \mathrm{Me}\right)$ 的 $\mathrm{R}^{2}$ 取代基也能以中等产率得到目标产
物. 目标产物(2q, 2r)的产率分别为 $61 \%$ 和 $42 \%$. 此外, 还尝试了 $\mathrm{R}^{2}$ 是䒺环、甲基、环丙基的底物 $(1 \mathrm{~s} \sim 1 \mathrm{u})$, 反 应能以 $87 \% \sim 98 \%$ 的产率顺利转化为相应的产物( $2 \mathrm{~s} \sim$

2u).

\section{3 可能的反应机理}

为了进一步了解该反应的可能机理，做了相关的实 验. 首先, 通过循环伏安法 $(\mathrm{CV})$ 研究了反应物的氧化还 原能力. 如图 1 所示, 可以观察到 TBAI 的两个氧化 峰 ${ }^{[10]}$ 分别在 $0.91,1.58 \mathrm{~V}$ (vs Ag/AgCl, Curve b), 分别对 应 TBAI, 经历从 $\mathrm{I}^{-}$到 $\mathrm{I}_{3}^{-}$和从 $\mathrm{I}_{3}^{-}$到 $\mathrm{I}_{2}$ 的两次氧化过 程 ${ }^{[11]}$. 化合物 $1 \mathrm{a}$ 在体系中的氧化峰在 $1.0 \mathrm{~V}$ (vs $\mathrm{Ag} / \mathrm{AgCl}$, Curve c). 曲线 $\mathrm{d}$ 中的氧化峰 $(E=0.85,1.58 \mathrm{~V}$ vs $\mathrm{Ag} / \mathrm{AgCl}$; Curve d) 相较于曲线 $\mathrm{b}$ 要明显更小. 以上结 果表明, TBAI 先被阳极氧化且作为碘源参与了反应. 如 
Scheme 2 所示, 往模型反应中添加过量的自由基捕获剂 2,2,6,6-四甲基哌啶氧化物(TEMPO)或 1,1-二苯乙烯后, 该反应仍分别以 $79 \%$ 和 $83 \%$ 的产率顺利进行. 该结果表 明反应可能不涉及自由基机理. 当采用无碘电解质时, 反应无法向 2a 顺利转化. 综上所述，同文献[12]报道一 致, 碘盐具有电解质、催化剂和碘源三重角色, 该反应 可能是通过阳极氧化原位生成的碘正离子活化炔烃促 进分子内亲核加成的发生 ${ }^{[13]}$.

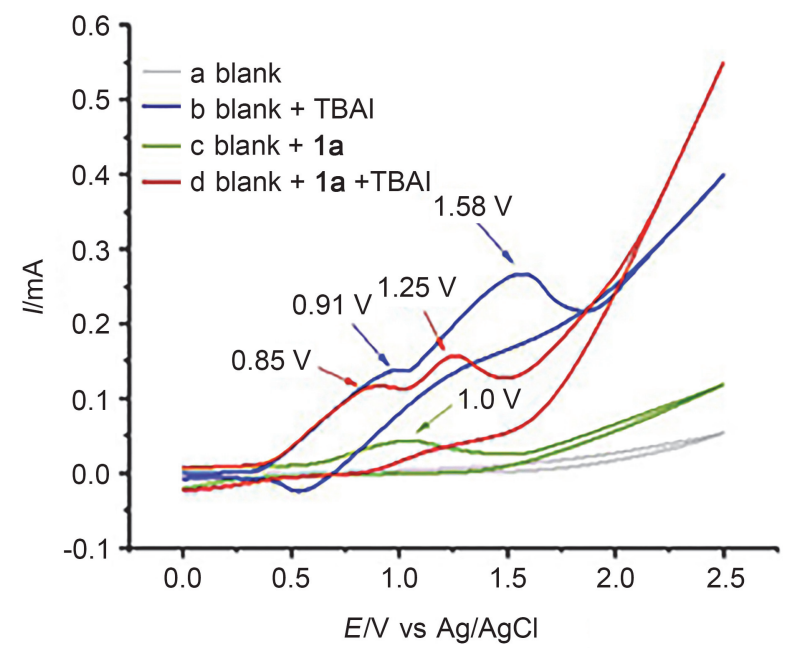

图 1 循环伏安法

Figure 1 Cyclic voltammetry

Cyclic voltammograms using glassy carbon working electrode, platinum wire counter electrode, and $\mathrm{Ag} / \mathrm{AgCl}$ (in saturated $\mathrm{KCl}$ solution) as the reference electrode on $\mathrm{CHI} 600 \mathrm{E}$ station at a scan rate of $100 \mathrm{mV} / \mathrm{s}$. Curve a (blank): $2 \mathrm{mmol} / \mathrm{L} \mathrm{KPF}_{6}$ in $\mathrm{CH}_{3} \mathrm{CN} / \mathrm{H}_{2} \mathrm{O}(6 \mathrm{~mL}, V: V=5 / 1)$. Curve b (blank + TBAI): $75 \mathrm{mmol} / \mathrm{L}$ TBAI and $2 \mathrm{mmol} / \mathrm{L} \mathrm{KPF}_{6}$ in $\mathrm{CH}_{3} \mathrm{CN} / \mathrm{H}_{2} \mathrm{O}(6 \mathrm{~mL}, V: V=5 / 1)$. Curve c (blank $\left.+1 \mathbf{a}\right): 50 \mathrm{mmol} / \mathrm{L}$ 1a and $2 \mathrm{mmol} / \mathrm{L} \mathrm{KPF}{ }_{6}$ in $\mathrm{CH}_{3} \mathrm{CN} / \mathrm{H}_{2} \mathrm{O}(6 \mathrm{~mL}, V: V=5 / 1)$. Curve $\mathrm{d}$ (blank + 1a+TBAI): $50 \mathrm{mmol} / \mathrm{L} 1 \mathrm{a}, 75 \mathrm{mmol} / \mathrm{L}$ TBAI and $2 \mathrm{mmol} / \mathrm{L}$ $\mathrm{KPF}_{6}$ in $\mathrm{CH}_{3} \mathrm{CN} / \mathrm{H}_{2} \mathrm{O}(6 \mathrm{~mL}, V: V=5 / 1)$.<smiles>Nc1ccccc1C#Cc1ccccc1</smiles>
Standard conditions<smiles>CC(C)Cn1c(-c2ccccc2)c(I)c2ccccc21</smiles><smiles>C(#Cc1ccccc1Nc1ccccc1)c1ccccc1</smiles>

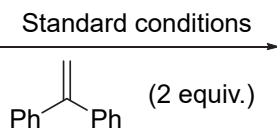<smiles>[13CH2]n1c(-c2ccccc2)c(I)c2ccccc21</smiles><smiles>C(#Cc1ccccc1Nc1ccccc1)c1ccccc1</smiles>
$\frac{\text { Standard conditions }}{\mathrm{BAPF}_{6} \text { instead of TBAI }}$<smiles>Cn1c(-c2ccccc2)c(I)c2ccccc21</smiles>
not detected

图式 2 控制实验

Scheme 2 Control experiments

根据上述结果和已报道的文献 ${ }^{[2-3,12-13]}$, 提出了以下
可能的机理(Scheme 3): 首先是碘负离子在电极阳极原 位生成碘正离子. 碘正离子活化邻炔基苯胺 $1 \mathrm{a}$ 中炔烃 官能团，形成碘鎓离子中间体 I. 随后氮的分子内亲核 进攻导致闭环，生成吲哚中间体 II. 中间体 II 失去一个 质子, 最终获得目标产物 $\mathbf{2 a}$. 同时, 水在阴极还原, 生 成氢气和氢氧根离子.

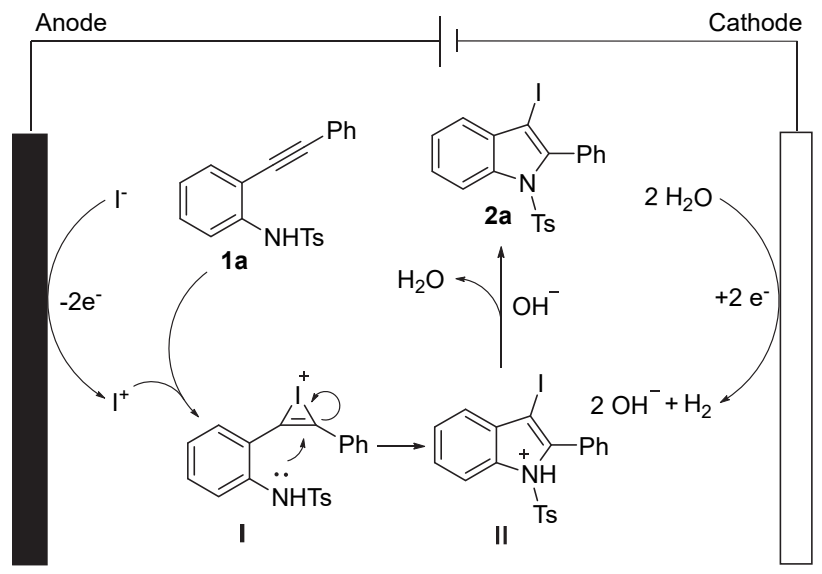

图式 3 可能的反应机理

Scheme 3 Proposed reaction mechanism

\section{2 结论}

从容易获得的邻炔基苯胺出发, 电化学氧化的碘盐 作为氧化还原催化剂和碘源, 实现了 3-碘吲哚骨架的构 建. 该反应条件温和, 无需额外添加氧化剂和碱, 底物 适用范围广，操作简单，是一种代替有机高价碘的绿色 合成策略.

\section{3 实验部分}

\section{1 仪器与试剂}

电源是国产双显示直流恒压电源 (UTP1303/ UTP3305)，阳极和阴极均为铂片 $(1.0 \mathrm{~cm} \times 1.0 \mathrm{~cm} \times 0.2$ $\mathrm{mm})$, 这些电极购买自中国上海. 质谱通过一台带有 Agilent 5975 质子选择器的 Agilent 7890 气相色谱仪测 定; 薄层色谱法(TLC)使用默克硅胶板(60F-254); 循环 伏安法 $(\mathrm{CV})$ 通过上海晨华 CHI600E 型电化学工作站测 定; 熔点由 OptiMelt MPA 100 测定; 液相产率以 1-硝基 萗为内标使用 $254 \mathrm{~nm} \mathrm{UV}$ 检测器的高效液相色谱仪 (HPLC) 测定; 柱层析分离以 200 300 目硅胶为固定相, 以石油醚 $(\mathrm{PE}) /$ 乙酸乙酯 $(\mathrm{EA})$ 混合物为洗脱剂; 高分辨 质谱(HRMS)由 ThermoFisher LTQ Orbitrap X 液相-质谱 联用仪测定; ${ }^{1} \mathrm{H}$ NMR 和 ${ }^{13} \mathrm{C}$ NMR 由 Bruker DRX-600 和 AMX-400 型核磁共振仪测定, 化学位移是以 $\delta$ 表示 的, 参考了 $\mathrm{CDCl}_{3}$ 的溶剂峰, 氢谱、碳谱分别定标在 $\delta$ $7.26\left({ }^{1} \mathrm{H}\right.$ NMR $), 77.16\left({ }^{13} \mathrm{C}\right.$ NMR $)$, DMSO- $d_{6}$ 的溶剂峰碳 谱定标在 $\delta 39.52\left({ }^{13} \mathrm{C} \mathrm{NMR}\right)$. 化合物 $\mathbf{1}^{[14]}$ 根据已报道的 
文献制备. 所有溶剂在使用前都是从适当的干燥剂中蒸 馏出来的, 所用试剂均为市售, 直接使用, 没有进一步 纯化处理.

\section{2 实验方法}

向 $10 \mathrm{~mL}$ 干燥三口烧瓶中依次加入邻炔基苯胺 1 (0.3 mmol), TBAI (0.45 mmol ), $\mathrm{KPF}_{6}(0.75 \mathrm{mmol})$, 然后 加入混合溶剂 $\mathrm{CH}_{3} \mathrm{CN} / \mathrm{H}_{2} \mathrm{O}(6 \mathrm{~mL}, V / V=5 / 1)$, 紧接着插 入两个铂片电极 $(1.0 \mathrm{~cm} \times 1.0 \mathrm{~cm} \times 0.2 \mathrm{~mm})$ 分别作为反 应的阳极和阴极. 反应搅拌 $10 \sim 15 \mathrm{~min}$ 保证均相体系. 接通电源, 将电流调至 $3 \mathrm{~mA}$ 恒定电流. 通过 TLC 监控, 直至原料完全消耗, 停止反应 $(24 \mathrm{~h})$. 将析出的产物过 滤, 石油醚洗涤, 然后滤液用乙酸乙酯萃取, 饱和食盐 水洗涤, 无水硫酸钠干燥, 在减压下除掉溶剂, 剩余粗 产物利用 $200 \sim 300$ 目硅胶, 石油醚和乙酸乙酯的混合 物 $(V / V=10 / 1)$ 为洗脱剂的柱层析提纯, 合并滤饼获得纯 净的目标产物 $\mathbf{2 a} \sim \mathbf{2 u}$.

3-碘-2-苯基-1-甲苯磺酰基-1H-吲哚(2a)：白色固体， $133.5 \mathrm{mg}$, 产率 94\%. m.p. $121 \sim 122{ }^{\circ} \mathrm{C}$ (文献值 ${ }^{[3 \mathrm{c}]}$ : m.p. $\left.113 \sim 115{ }^{\circ} \mathrm{C}\right) ;{ }^{1} \mathrm{H}$ NMR (600 MHz, $\left.\mathrm{CDCl}_{3}\right) \delta: 8.35$ (d, $J=$ $8.4 \mathrm{~Hz}, 1 \mathrm{H}), 7.54 \sim 7.43(\mathrm{~m}, 5 \mathrm{H}), 7.42 \sim 7.37(\mathrm{~m}, 3 \mathrm{H}), 7.35$ (d, $J=8.4 \mathrm{~Hz}, 2 \mathrm{H}), 7.10$ (d, $J=8.2 \mathrm{~Hz}, 2 \mathrm{H}), 2.32$ (s, 3H).

3-碘-5-甲基-2-苯基-1-甲苯磺酰基- $1 H$-吲哚(2b): 白 色固体, $106.7 \mathrm{mg}$, 产率 $73 \%$. m.p. 101 $102{ }^{\circ} \mathrm{C}$ (文献 值 ${ }^{[3 \mathrm{c}]}$ : m.p. $\left.95 \sim 97{ }^{\circ} \mathrm{C}\right) ;{ }^{1} \mathrm{H}$ NMR $\left(600 \mathrm{MHz}, \mathrm{CDCl}_{3}\right) \delta$ : $8.18(\mathrm{~d}, J=8.5 \mathrm{~Hz}, 1 \mathrm{H}), 7.51 \sim 7.45(\mathrm{~m}, 3 \mathrm{H}), 7.38(\mathrm{~d}$, $J=8.3 \mathrm{~Hz}, 2 \mathrm{H}), 7.32(\mathrm{~d}, J=8.3 \mathrm{~Hz}, 2 \mathrm{H}), 7.26(\mathrm{~m}, 1 \mathrm{H})$, 7.20 (s, 1H), 7.09 (d, J=8.0 Hz, 2H), 2.48 (s, 3H), 2.32 (s, $3 \mathrm{H})$.

3-碘-5-甲氧基-2-苯基-1-甲苯磺酰基- $1 H$-吲哚(2c): 白色固体, $122.3 \mathrm{mg}$, 产率 $81 \%$. m.p. 142 $143{ }^{\circ} \mathrm{C}$ (文献 值 ${ }^{[3 \mathrm{c}]}$ : m.p. $\left.70 \sim 72{ }^{\circ} \mathrm{C}\right) ;{ }^{1} \mathrm{H}$ NMR $\left(600 \mathrm{MHz}, \mathrm{CDCl}_{3}\right) \delta$ : $8.19(\mathrm{~d}, J=9.1 \mathrm{~Hz}, 1 \mathrm{H}), 7.51 \sim 7.44(\mathrm{~m}, 3 \mathrm{H}), 7.38$ (d, $J=$ $6.7 \mathrm{~Hz}, 2 \mathrm{H}), 7.29$ (d, $J=8.4 \mathrm{~Hz}, 2 \mathrm{H}), 7.08$ (d, $J=8.0 \mathrm{~Hz}$, 2H), $7.03(\mathrm{dd}, J=9.1,2.6 \mathrm{~Hz}, 1 \mathrm{H}), 6.84(\mathrm{~d}, J=2.6 \mathrm{~Hz}$, 1H), 3.89 (s, 3H), 2.32 (s, 3H).

3-碘-5-氟-2-苯基-1-甲苯磺酰基- $1 H$-吲哚(2d): 白色 固体， $109.1 \mathrm{mg}$, 产率 $74 \%$. m.p. $120 \sim 121{ }^{\circ} \mathrm{C}$ (文献 值 ${ }^{[15 a]}$ : m.p. $\left.122{ }^{\circ} \mathrm{C}\right) ;{ }^{1} \mathrm{H}$ NMR (600 MHz, $\left.\mathrm{CDCl}_{3}\right) \delta: 8.29$ $(\mathrm{dd}, J=9.1,4.4 \mathrm{~Hz}, 1 \mathrm{H}), 7.51(\mathrm{t}, J=7.3 \mathrm{~Hz}, 1 \mathrm{H}), 7.47$ (t, $J=7.4 \mathrm{~Hz}, 2 \mathrm{H}), 7.37$ (d, $J=7.1 \mathrm{~Hz}, 2 \mathrm{H}), 7.30$ (d, $J=8.3$ $\mathrm{Hz}, 2 \mathrm{H}), 7.16(\mathrm{td}, J=9.0,2.6 \mathrm{~Hz}, 1 \mathrm{H}), 7.13 \sim 7.08(\mathrm{~m}$, $3 \mathrm{H}), 2.33$ (s, 3H).

3-碘-5-氯-2-苯基-1-甲苯磺酰基- $1 H$-吲哚(2e): 白色 固体, $99.1 \mathrm{mg}$, 产率 $65 \%$. m.p. $128 \sim 129{ }^{\circ} \mathrm{C}$ (文献值 ${ }^{[3 \mathrm{c}]}$ : m.p. $\left.129 \sim 131{ }^{\circ} \mathrm{C}\right) ;{ }^{1} \mathrm{H}$ NMR $\left(600 \mathrm{MHz}, \mathrm{CDCl}_{3}\right) \delta: 8.26$ $(\mathrm{d}, J=8.8 \mathrm{~Hz}, 1 \mathrm{H}), 7.52(\mathrm{t}, J=7.4 \mathrm{~Hz}, 1 \mathrm{H}), 7.47(\mathrm{t}, J=7.4$ $\mathrm{Hz}, 2 \mathrm{H}), 7.43 \sim 7.39(\mathrm{~m}, 2 \mathrm{H}), 7.36(\mathrm{~d}, J=7.1 \mathrm{~Hz}, 2 \mathrm{H})$, 7.31 (d, $J=8.3 \mathrm{~Hz}, 2 \mathrm{H}), 7.11$ (d, $J=8.2 \mathrm{~Hz}, 2 \mathrm{H}), 2.34$ (s, $3 \mathrm{H})$.

3-碘-5-溴-2-苯基-1-甲苯磺酰基- $1 H$-吲哚(2f $)^{[3 c]}$ : 白 色固体, $139.2 \mathrm{mg}$, 产率 $84 \%$. m.p. $129 \sim 130{ }^{\circ} \mathrm{C} ;{ }^{1} \mathrm{H}$ NMR (600 MHz, $\left.\mathrm{CDCl}_{3}\right) \delta: 8.21(\mathrm{~d}, J=8.9 \mathrm{~Hz}, 1 \mathrm{H}), 7.58$ $(\mathrm{d}, J=1.9 \mathrm{~Hz}, 1 \mathrm{H}), 7.55 \sim 7.50(\mathrm{~m}, 2 \mathrm{H}), 7.47(\mathrm{t}, J=7.4$ $\mathrm{Hz}, 2 \mathrm{H}), 7.36$ (d, $J=7.0 \mathrm{~Hz}, 2 \mathrm{H}), 7.30$ (d, $J=8.4 \mathrm{~Hz}, 2 \mathrm{H})$, $7.11(\mathrm{~d}, \quad J=8.2 \mathrm{~Hz}, 2 \mathrm{H}), 2.33(\mathrm{~s}, 3 \mathrm{H})$.

3-碘-5-羧酸甲酯-2-苯基-1-甲苯磺酰基- $1 H$-吲哚 (2g): 白色固体, $156.2 \mathrm{mg}$, 产率 98\%. m.p. 158 $159{ }^{\circ} \mathrm{C}$; ${ }^{1} \mathrm{H}$ NMR $\left(600 \mathrm{MHz}, \mathrm{CDCl}_{3}\right) \delta: 8.37(\mathrm{~d}, J=9.2 \mathrm{~Hz}, 1 \mathrm{H})$, $8.15 \sim 8.11(\mathrm{~m}, 2 \mathrm{H}), 7.51(\mathrm{t}, J=7.4 \mathrm{~Hz}, 1 \mathrm{H}), 7.46(\mathrm{t}, J=$ $7.4 \mathrm{~Hz}, 2 \mathrm{H}), 7.34$ (d, $J=7.0 \mathrm{~Hz}, 2 \mathrm{H}), 7.31$ (d, $J=8.4 \mathrm{~Hz}$, 2H), 7.10 (d, $J=8.1 \mathrm{~Hz}, 2 \mathrm{H}), 3.97$ (s, 3H), 2.32 (s, 3H); ${ }^{13} \mathrm{C} \mathrm{NMR}\left(150 \mathrm{MHz}, \mathrm{CDCl}_{3}\right) \delta: 167.0,145.5,142.5,139.7$, $135.0,132.2,131.8,131.1,129.7,129.7,127.7,127.2$, $127.0,126.7,124.4,115.9,75.5,52.4,21.7$; IR (KBr) $v$ : $3435,1709,1605,1434,545 \mathrm{~cm}^{-1}$. HRMS (ESI) calcd for $\mathrm{C}_{23} \mathrm{H}_{18} \mathrm{INNaO}_{4} \mathrm{~S}[\mathrm{M}+\mathrm{Na}]^{+}$553.9893, found 553.9886.

3-碘-5-氰基-2-苯基-1-甲苯磺酰基- $1 H$-吲哚(2h)：白 色固体，140.5 mg，产率 94\%. m.p. 186 $188{ }^{\circ} \mathrm{C}$ (文献 值 ${ }^{[2 \mathrm{c}]}$ : m.p. $\left.186 \sim 187{ }^{\circ} \mathrm{C}\right) ;{ }^{1} \mathrm{H} \mathrm{NMR}\left(600 \mathrm{MHz}, \mathrm{CDCl}_{3}\right) \delta$ : $8.43(\mathrm{~d}, J=8.7 \mathrm{~Hz}, 1 \mathrm{H}), 7.77(\mathrm{~s}, 1 \mathrm{H}), 7.68(\mathrm{~d}, J=8.7 \mathrm{~Hz}$, $1 \mathrm{H}), 7.52$ (t, $J=7.5 \mathrm{~Hz}, 1 \mathrm{H}), 7.45$ (t, $J=7.6 \mathrm{~Hz}, 2 \mathrm{H}), 7.29$ (d, $J=8.2 \mathrm{~Hz}, 4 \mathrm{H}), 7.13(\mathrm{~d}, J=8.2 \mathrm{~Hz}, 2 \mathrm{H}), 2.35$ (s, 3H).

3-碘-5-硝基-2-苯基-1-甲苯磺酰基- $1 H$-吲哚(2i): 黄 色固体, $116.6 \mathrm{mg}$, 产率 75\%. m.p. 196 $198{ }^{\circ} \mathrm{C} ;{ }^{1} \mathrm{H}$ NMR (600 MHz, $\left.\mathrm{CDCl}_{3}\right) \delta: 8.46(\mathrm{~d}, J=9.2 \mathrm{~Hz}, 1 \mathrm{H})$, $8.35 \sim 8.29(\mathrm{~m}, 2 \mathrm{H}), 7.53(\mathrm{t}, J=7.5 \mathrm{~Hz}, 1 \mathrm{H}), 7.46(\mathrm{t}, J=$ $7.6 \mathrm{~Hz}, 2 \mathrm{H}), 7.30(\mathrm{~m}, 4 \mathrm{H}), 7.14$ (d, $J=8.2 \mathrm{~Hz}, 2 \mathrm{H}), 2.36$ $(\mathrm{s}, 3 \mathrm{H}) ;{ }^{13} \mathrm{C}$ NMR $\left(150 \mathrm{MHz}, \mathrm{CDCl}_{3}\right) \delta: 146.0,145.0$, $144.2,140.1,134.8,132.4,131.7,130.5,130.0,129.9$, $127.8,127.2,120.9,118.5,116.4,74.5,21.8$; IR (KBr) $v$ : 3448, 1606, 1596, 1516, 1439, $544 \mathrm{~cm}^{-1}$. HRMS (ESI) calcd for $\mathrm{C}_{21} \mathrm{H}_{15} \mathrm{IN}_{2} \mathrm{NaO}_{4} \mathrm{~S}[\mathrm{M}+\mathrm{Na}]^{+}$540.9689, found 540.9675 .

3-碘-2-(4-甲氧基苯基)-1- 甲苯磺酰基- $1 H$-吲哚 (2j): 白色固体, $137.4 \mathrm{mg}$, 产率 91\%. m.p. 145 $146{ }^{\circ} \mathrm{C}$ (文献值 ${ }^{[3 c]}$ : m.p. $143 \sim 145{ }^{\circ} \mathrm{C}$ ); ${ }^{1} \mathrm{H}$ NMR (600 MHz, $\left.\mathrm{CDCl}_{3}\right) \delta: 8.31(\mathrm{~d}, J=8.4 \mathrm{~Hz}, 1 \mathrm{H}), 7.46 \sim 7.40(\mathrm{~m}, 2 \mathrm{H})$, 7.35 (dd, $J=17.3,7.8 \mathrm{~Hz}, 3 \mathrm{H}), 7.28$ (s, 4H), 7.09 (d, $J=$ $8.1 \mathrm{~Hz}, 2 \mathrm{H}), 2.48(\mathrm{~s}, 3 \mathrm{H}), 2.32(\mathrm{~s}, 3 \mathrm{H})$.

3-碘-2-(4-甲基苯基)-1-甲苯磺酰基- $1 H$-吲哚(2k): 
白色固体, $136.0 \mathrm{mg}$, 产率 93\%. m.p. 125 $126{ }^{\circ} \mathrm{C}$ (文献 值 ${ }^{[3 \mathrm{cc}}$ : m.p. $\left.125 \sim 127{ }^{\circ} \mathrm{C}\right) ;{ }^{1} \mathrm{H}$ NMR $\left(600 \mathrm{MHz}, \mathrm{CDCl}_{3}\right) \delta$ : $8.33(\mathrm{~d}, J=8.4 \mathrm{~Hz}, 1 \mathrm{H}), 7.47 \sim 7.41(\mathrm{~m}, 2 \mathrm{H}), 7.39 \sim 7.34$ (m, 3H), $7.30 \sim 7.29(\mathrm{~m}, 4 \mathrm{H}), 7.09(\mathrm{~d}, J=8.2 \mathrm{~Hz}, 2 \mathrm{H})$, $2.49(\mathrm{~s}, 3 \mathrm{H}), 2.32(\mathrm{~s}, 3 \mathrm{H})$.

3-碘-2-(4-叔丁基苯基)-1-甲苯磺酰基-1 $H$-吲哚(2I): 白色固体, $146.1 \mathrm{mg}$, 产率 92\%. m.p. $176 \sim 177{ }^{\circ} \mathrm{C} ;{ }^{1} \mathrm{H}$ NMR $\left(600 \mathrm{MHz}, \mathrm{CDCl}_{3}\right) \delta: 8.33(\mathrm{~d}, J=8.4 \mathrm{~Hz}, 1 \mathrm{H})$, $7.49 \sim 7.40(\mathrm{~m}, 4 \mathrm{H}), 7.36(\mathrm{t}, J=7.5 \mathrm{~Hz}, 1 \mathrm{H}), 7.31(\mathrm{~d}, J=$ $8.1 \mathrm{~Hz}, 4 \mathrm{H}), 7.07$ (d, $J=8.1 \mathrm{~Hz}, 2 \mathrm{H}), 2.31$ (s, 3H), 1.43 (s, $9 \mathrm{H}) ;{ }^{13} \mathrm{C}$ NMR $\left(150 \mathrm{MHz}, \mathrm{CDCl}_{3}\right) \delta: 152.3,144.9,141.3$, $137.1,135.1,132.4,131.5,129.5,128.4,127.0,126.0$, 124.6, 124.5, 122.2, 116.1, 75.6, 34.9, 31.5, 21.6; IR (KBr) $v: 3444,1597,1447,546 \mathrm{~cm}^{-1}$. HRMS (ESI) calcd for $\mathrm{C}_{25} \mathrm{H}_{24} \mathrm{INNaO}_{2} \mathrm{~S}[\mathrm{M}+\mathrm{Na}]^{+}$552.0465, found 552.0447.

3-碘-2-(4-氟苯基)-1-甲苯磺酰基- $1 H$-吲哚(2m): 白 色固体, $131.2 \mathrm{mg}$, 产率 $89 \%$. m.p. $154 \sim 155{ }^{\circ} \mathrm{C}$ (文献 值 ${ }^{[3 \mathrm{cc}}$ : m.p. $\left.183 \sim 185{ }^{\circ} \mathrm{C}\right) ;{ }^{1} \mathrm{H}$ NMR $\left(400 \mathrm{MHz}, \mathrm{CDCl}_{3}\right) \delta$ : $8.34(\mathrm{~d}, J=8.3 \mathrm{~Hz}, 1 \mathrm{H}), 7.50 \sim 7.30(\mathrm{~m}, 7 \mathrm{H}), 7.17$ (t, $J=$ $8.4 \mathrm{~Hz}, 2 \mathrm{H}), 7.11(\mathrm{~d}, J=7.9 \mathrm{~Hz}, 2 \mathrm{H}), 2.32(\mathrm{~s}, 3 \mathrm{H}) ;{ }^{13} \mathrm{C}$ NMR $\left(100 \mathrm{MHz}, \mathrm{CDCl}_{3}\right) \delta: 163.33(\mathrm{~d}, J=249.6 \mathrm{~Hz})$, 162.1, 145.3, 140.0, 137.1, 135.1, 133.77 (d, $J=8.5 \mathrm{~Hz})$, 132.2, 129.6, 127.54 (d, $J=3.3 \mathrm{~Hz}), 126.9,126.3,124.8$, 122.3, 116.0, 114.83 (d, $J=21.8 \mathrm{~Hz}), 76.2,21.7$.

3-碘-2-(4-氯苯基)-1-甲苯磺酰基- $1 H$-吲哚(2n): 白 色固体, $128.0 \mathrm{mg}$, 产率 $84 \%$. m.p. $168 \sim 169{ }^{\circ} \mathrm{C}$ (文献 值 ${ }^{[3 \mathrm{c}]}$ : m.p. $\left.168 \sim 170{ }^{\circ} \mathrm{C}\right) ;{ }^{1} \mathrm{H}$ NMR $\left(600 \mathrm{MHz}, \mathrm{CDCl}_{3}\right) \delta$ : $8.31(\mathrm{~d}, J=8.4 \mathrm{~Hz}, 1 \mathrm{H}), 7.48 \sim 7.40(\mathrm{~m}, 4 \mathrm{H}), 7.38(\mathrm{t}, J=$ $7.5 \mathrm{~Hz}, 1 \mathrm{H}), 7.32(\mathrm{~d}, J=8.0 \mathrm{~Hz}, 4 \mathrm{H}), 7.10(\mathrm{~d}, J=8.1 \mathrm{~Hz}$, $2 \mathrm{H}), 2.33$ (s, 3H).

3-碘-2-(3-氯苯基)-1-甲苯磺酰基- $1 H$-吲哚(2o): 白 色固体, $144.7 \mathrm{mg}$, 产率 95\%. m.p. $115 \sim 116{ }^{\circ} \mathrm{C} ;{ }^{1} \mathrm{H}$ NMR $\left(600 \mathrm{MHz}, \mathrm{CDCl}_{3}\right) \delta: 8.32(\mathrm{~d}, J=8.4 \mathrm{~Hz}, 1 \mathrm{H})$, $7.49 \sim 7.45(\mathrm{~m}, 2 \mathrm{H}), 7.40(\mathrm{~m}, 3 \mathrm{H}), 7.33(\mathrm{t}, J=9.3 \mathrm{~Hz}, 3 \mathrm{H})$, $7.23(\mathrm{t}, J=1.8 \mathrm{~Hz}, 1 \mathrm{H}), 7.12(\mathrm{~d}, J=8.1 \mathrm{~Hz}, 2 \mathrm{H}), 2.34$ (s, $3 \mathrm{H}) ;{ }^{13} \mathrm{C}$ NMR $\left(150 \mathrm{MHz}, \mathrm{CDCl}_{3}\right) \delta: 145.4,139.5,137.1$, $135.0,133.5,133.3,132.1,131.7,130.2,129.7,129.5$, 129.0, 127.0, 126.5, 124.9, 122.5, 116.0, 76.3, 21.7; IR (KBr) $v$ : $3440,1598,1446,1377,544 \mathrm{~cm}^{-1}$. HRMS (ESI) calcd for $\mathrm{C}_{21} \mathrm{H}_{15} \mathrm{ClINNaO}_{2} \mathrm{~S}[\mathrm{M}+\mathrm{Na}]^{+}$529.9449, found 529.9437.

3-碘-2-(4-溴苯基)-1-甲苯磺酰基- $1 H$-吲梠(2p)：白 色固体, $134.2 \mathrm{mg}$, 产率 $81 \%$. m.p. $174 \sim 176{ }^{\circ} \mathrm{C} ;{ }^{1} \mathrm{H}$ NMR (400 MHz, $\left.\mathrm{CDCl}_{3}\right) \delta: 8.33(\mathrm{~d}, J=8.3 \mathrm{~Hz}, 1 \mathrm{H}), 7.62$ (d, $J=8.2 \mathrm{~Hz}, 2 \mathrm{H}), 7.51 \sim 7.38(\mathrm{~m}, 3 \mathrm{H}), 7.34(\mathrm{~d}, J=8.1$
Hz, 2H), 7.28 (d, $J=7.8 \mathrm{~Hz}, 2 \mathrm{H}), 7.13$ (d, $J=8.0 \mathrm{~Hz}, 2 \mathrm{H})$, $2.35(\mathrm{~s}, 3 \mathrm{H}) ;{ }^{13} \mathrm{C}$ NMR $\left(100 \mathrm{MHz}, \mathrm{CDCl}_{3}\right) \delta: 145.3,139.8$, $137.0,134.9,133.3,132.3,130.9,130.5,129.6,126.8$, 126.4, 124.9, 123.9, 122.3, 116.0, 76.3, 21.6; IR (KBr) v: $3440,1597,1468,1369,542 \mathrm{~cm}^{-1}$. HRMS (ESI) calcd for $\mathrm{C}_{21} \mathrm{H}_{15} \mathrm{BrINNaO}_{2} \mathrm{~S}[\mathrm{M}+\mathrm{Na}]^{+}$573.8944, found 573.8956.

3-碘-2-(4-氧基苯基)-1-甲苯磺酰基- $1 H$-吲哚(2q): 白色固体, $91.2 \mathrm{mg}$, 产率 61\%. m.p. 197 $199{ }^{\circ} \mathrm{C} ;{ }^{1} \mathrm{H}$ NMR $\left(600 \mathrm{MHz}, \mathrm{CDCl}_{3}\right) \delta: 8.29(\mathrm{~d}, J=8.4 \mathrm{~Hz}, 1 \mathrm{H}), 7.75$ (d, $J=8.1 \mathrm{~Hz}, 2 \mathrm{H}), 7.55(\mathrm{~d}, J=8.1 \mathrm{~Hz}, 2 \mathrm{H}), 7.48$ (t, $J=7.7$ $\mathrm{Hz}, 1 \mathrm{H}), 7.42(\mathrm{~d}, J=7.1 \mathrm{~Hz}, 1 \mathrm{H}), 7.40 \sim 7.37(\mathrm{~m}, 1 \mathrm{H})$, $7.31(\mathrm{~d}, J=8.3 \mathrm{~Hz}, 2 \mathrm{H}), 7.11(\mathrm{~d}, J=8.3 \mathrm{~Hz}, 2 \mathrm{H}), 2.32$ (s, $3 \mathrm{H}) ;{ }^{13} \mathrm{C}$ NMR (150 MHz, DMSO- $\left.d_{6}\right) \delta$ : 145.8, 139.1, $136.5,136.3,133.2,132.2,132.1,131.6,130.1,126.9$, $126.5,125.3,122.3,118.6,115.6,111.7,78.9,39.7,39.5$, 39.4, 21.0; IR (KBr) v: 3224, 2228, 1607, 1445, $541 \mathrm{~cm}^{-1}$. HRMS (ESI) calcd for $\mathrm{C}_{22} \mathrm{H}_{15} \mathrm{IN}_{2} \mathrm{NaO}_{2} \mathrm{~S}[\mathrm{M}+\mathrm{Na}]^{+}$ 573.8944, found 573.8956 .

3-碘-2-(4-羞酸甲酯基苯基)-1-甲苯磺酰基- $1 H$-吲哚 (2r): 白色固体, $67.0 \mathrm{mg}$, 产率 $42 \%$. m.p. $183 \sim 185{ }^{\circ} \mathrm{C}$; ${ }^{1} \mathrm{H}$ NMR $\left(600 \mathrm{MHz}, \mathrm{CDCl}_{3}\right) \delta: 8.30(\mathrm{~d}, J=8.4 \mathrm{~Hz}, 1 \mathrm{H})$, $8.14(\mathrm{~d}, J=8.2 \mathrm{~Hz}, 2 \mathrm{H}), 7.47$ (dd, $J=12.2,8.2 \mathrm{~Hz}, 3 \mathrm{H}$ ), $7.42(\mathrm{~d}, J=7.4 \mathrm{~Hz}, 1 \mathrm{H}), 7.38(\mathrm{t}, J=7.5 \mathrm{~Hz}, 1 \mathrm{H}), 7.31(\mathrm{~d}$, $J=8.3 \mathrm{~Hz}, 2 \mathrm{H}), 7.10$ (d, $J=8.2 \mathrm{~Hz}, 2 \mathrm{H}), 3.98$ (s, 3H), 2.32 (s, 3H); ${ }^{13} \mathrm{C}$ NMR $\left(150 \mathrm{MHz}, \mathrm{CDCl}_{3}\right) \delta: 166.9,145.4$, $140.0,137.2,136.3,134.9,132.4,131.9,130.8,129.7$, $128.9,126.9,126.6,125.0,122.5,116.2,76.7,52.4,21.7$; IR (KBr) $v: 3435,1714,1613,1597,541 \mathrm{~cm}^{-1}$. HRMS (ESI) calcd for $\mathrm{C}_{23} \mathrm{H}_{18} \mathrm{INNaO} \mathrm{NN}_{4} \mathrm{~S}[\mathrm{M}+\mathrm{Na}]^{+}$553.8993, found 553.8977 .

3-碘-2-(芸-1-基)-1-甲苯磺酰基- $1 H$-吲哚(2s)：白色 固体, $153.9 \mathrm{mg}$, 产率 98\%. m.p. $166 \sim 168{ }^{\circ} \mathrm{C} ;{ }^{1} \mathrm{H}$ NMR $\left(600 \mathrm{MHz}, \mathrm{CDCl}_{3}\right) \delta: 8.47(\mathrm{~d}, J=8.3 \mathrm{~Hz}, 1 \mathrm{H}), 8.05(\mathrm{~d}, J=$ $8.2 \mathrm{~Hz}, 1 \mathrm{H}), 7.94(\mathrm{~d}, J=8.2 \mathrm{~Hz}, 1 \mathrm{H}), 7.64 \sim 7.59(\mathrm{~m}, 1 \mathrm{H})$, $7.56(\mathrm{t}, J=8.0 \mathrm{~Hz}, 2 \mathrm{H}), 7.52 \sim 7.45(\mathrm{~m}, 3 \mathrm{H}), 7.32(\mathrm{t}, J=$ $7.0 \mathrm{~Hz}, 2 \mathrm{H}), 7.28(\mathrm{~d}, J=8.3 \mathrm{~Hz}, 2 \mathrm{H}), 6.97(\mathrm{~d}, J=8.1 \mathrm{~Hz}$, 2H), $2.29(\mathrm{~s}, 3 \mathrm{H}) ;{ }^{13} \mathrm{C}$ NMR $\left(150 \mathrm{MHz}, \mathrm{CDCl}_{3}\right) \delta: 145.0$, $139.1,137.0,135.3,133.2,132.9,131.8,130.7,130.3$, $129.4,129.2,128.3,127.1,126.5,126.2,125.9,125.9$, 124.6, 124.4, 122.2, 115.5, 76.5, 21.6; IR (KBr) v: 3450, $1595,544 \mathrm{~cm}^{-1}$. HRMS (ESI) calcd for $\mathrm{C}_{25} \mathrm{H}_{18} \mathrm{INNaO}_{2} \mathrm{~S}$ $[\mathrm{M}+\mathrm{Na}]^{+}$545.9995, found 545.9978.

3-碘-2-甲基-1-甲苯磺酰基- $1 H$-吲哚(2t): 白色固体, $107.3 \mathrm{mg}$, 产率 $87 \%$. m.p. $157 \sim 158{ }^{\circ} \mathrm{C}$ (文献值 ${ }^{[15 b]}$ : m.p. $\left.158 \sim 159{ }^{\circ} \mathrm{C}\right) ;{ }^{1} \mathrm{H}$ NMR $\left(600 \mathrm{MHz}, \mathrm{CDCl}_{3}\right) \delta: 8.18(\mathrm{~d}$, 
$J=8.3 \mathrm{~Hz}, 1 \mathrm{H}), 7.68(\mathrm{~d}, J=8.3 \mathrm{~Hz}, 2 \mathrm{H}), 7.35 \sim 7.28(\mathrm{~m}$,

$3 \mathrm{H}), 7.20$ (d, $J=8.2 \mathrm{~Hz}, 2 \mathrm{H}), 2.72$ (s, 3H), 2.33 (s, 3H).

3-碘-2-环丙基-1-甲苯磺酰基- $1 H$-吲哚(2u): 白色固

体, $116.8 \mathrm{mg}$, 产率 89\%. m.p. 91 92 ${ }^{\circ} \mathrm{C}$ (文献值 ${ }^{[2 c]}$ : m.p. $\left.89 \sim 90{ }^{\circ} \mathrm{C}\right) ;{ }^{1} \mathrm{H}$ NMR $\left(600 \mathrm{MHz}, \mathrm{CDCl}_{3}\right) \delta: 8.19(\mathrm{~d}, J=$ $9.2 \mathrm{~Hz}, 1 \mathrm{H}), 7.67(\mathrm{~d}, J=8.4 \mathrm{~Hz}, 2 \mathrm{H}), 7.38 \sim 7.34(\mathrm{~m}, 2 \mathrm{H})$, $7.29(\mathrm{t}, J=7.5 \mathrm{~Hz}, 1 \mathrm{H}), 7.18(\mathrm{~d}, J=8.2 \mathrm{~Hz}, 2 \mathrm{H}), 2.33$ (s, $3 \mathrm{H}), 2.04(\mathrm{~m}, 1 \mathrm{H}), 1.18 \sim 1.05(\mathrm{~m}, 2 \mathrm{H}), 1.01 \sim 0.89(\mathrm{~m}$, $2 \mathrm{H})$.

辅助材料(Supporting Information) 条件优化表格, 3碘吲哚的合成过程, 反应不兼容的底物，化合物 $\mathbf{2 a} \sim \mathbf{2 u}$ 的 ${ }^{1} \mathrm{H}$ NMR 和化合物 $\mathbf{2 g}, \mathbf{2 i}, \mathbf{2 l}, \mathbf{2 m}, \mathbf{2 0} \sim \mathbf{2 s}$ 的 ${ }^{13} \mathrm{C} \mathrm{NMR}$ 图谱. 这些材料可以免费从本刊网站(http://sioc-journal. $\mathrm{cn} /$ )上下载.

\section{References}

[1] (a) Sravanthi, T. V.; Manju, S. L. Eur. J. Pharm. Sci. 2016, 91, 1 (b) Holman, K. R.; Stanko, A. M.; Reisman, S. E. Chem. Soc. Rev. 2021, 50, 7891 .

(c) Kochanowska-Karamyan, A. J.; Hamann, M. T. Chem. Rev. 2010, 110, 4489 .

(d) Yu, R.; Fang, X. Chin. J. Org. Chem. 2021, 41, 2532 (in Chinese)

(余融融, 方显杰, 有机化学, 2021, 41, 2532.)

(e) Wang, R.; Xu, L.; Lu, Y.; Jiang, B.; Hao, W. Chin. J. Org. Chem. 2021, 41, 1582 (in Chinese)

(王榕，徐立晨，卢逸，姜波，郝文娟，有机化学, 2021，41, 1582.) (f) Gao, Z.; Wang, Y.; Song, H.; Xu, Z.; Jia, Y. Chin. J. Org. Chem. 2021, 41, 3126 (in Chinese)

(高中润, 王媛, 宋航, 徐正仁, 贾彦兴, 有机化学, 2021, 41, 3126.)

(g) Li, P.; Zhou, Z.; Yang, F.; Xu, Y.; Zhang, X. Chin. J. Org. Chem. 2021, 41, 3235 (in Chinese).

(李萍, 周志强, 杨帆, 徐瑶, 张小祥, 有机化学, 2021, 41, 3235.)

[2] (a) Yue, D.; Larock, R. C. Org. Lett. 2004, 6, 1037.

(b) Zhang, C.-P.; Wang, Z.-L.; Chen, Q.-Y.; Zhang, C.-T.; Gu, Y.-C.; Xiao, J.-C. Angew. Chem. Int. Ed. 2011, 50, 1896.

(c) Al-Saedy, M. A. E.; Harrity, J. P. A. Synlett. 2016, 27, 1674

(d) Chintawar, C. C.; Yadav, A. K.; Patil, N. T. Angew. Chem. Int. Ed. 2020, 59, 11808.

(e) Campo, M. A.; Larock, R. C. J. Org. Chem. 2002, 67, 5616

[3] (a) Barluenga, J.; Trincado, M.; Rubio, E.; González, J. M. Angew. Chem. Int. Ed. 2003, 42, 2406.

(b) Amjad, M.; Knight, D. W. Tetrahedron Lett. 2004, 45, 539.

(c) Li, Y.-L.; Li, J.; Yu, S.-N.; Wang, J.-B.; Yu, Y.-M.; Deng, J. Tetrahedron 2015, 71, 8271.

[4] (a) Novaes, L. F. T.; Liu, J.; Shen, Y.; Lu, L.; Meinhardt, J. M.; Lin, S. Chem. Soc. Rev. 2021, 50, 7941.

(b) Liu, J.; Lu, L.; Wood, D.; Lin, S. ACS Cent. Sci. 2020, 6, 1317.

(c) Pollok, D.; Waldvogel, S. R. Chem. Sci. 2020, 11, 12386.

(d) Little, R. D. J. Org. Chem. 2020, 85, 13375.

(e) Kingston, C.; Palkowitz, M. D.; Takahira, Y.; Vantourout, J. C.; Peters, B. K.; Kawamata, Y.; Baran, P. S. Acc. Chem. Res. 2020, 53, 72 .

(f) Wang, X.; Xu, X.; Wang, Z.; Fang, P.; Mei, T. Chin. J. Org. Chem. 2020, 40, 3738 (in Chinese).

(王向阳, 徐学涛, 王振华, 方萍, 梅天胜, 有机化学, 2020, 40, 3738.)

(g) Xiong, P.; Xu, H.-C. Acc. Chem. Res. 2019, 52, 3339 (h) Yuan, Y.; Lei, A. Acc. Chem. Res. 2019, 52, 3309.

(i) Wiebe, A.; Gieshoff, T.; Möhle, S.; Rodrigo, E.; Zirbes, M.; Waldvogel, S. R. Angew. Chem. Int. Ed. 2018, 57, 5594.

(j) Yan, M.; Kawamata, Y.; Baran, P. S. Chem. Rev. 2017, 117, 13230

(k) Francke, R.; Little, R. D. Chem. Soc. Rev. 2014, 43, 2492.

(1) Kingston, C.; Palkowitz, M. D.; Takahira, Y.; Vantourout, J. C.; Peters, B. K.; Kawamata, Y.; Baran, P. S. Acc. Chem. Res. 2020, 53, 72.

(m) Huang, C.; Li, Z.-Y.; Song, J.; Xu, H.-C. Angew. Chem. Int. Ed 2021, 60, 11237

(n) Kai, S.; Xiao, F.; Yu, B.; He, W.-M. Chin. J. Catal. 2021, 42, 1921.

(o) Liu, F.; Dai, J.; Cheng, X. Chin. J. Org. Chem. 2021, 41, 4014 (in Chinese)

(刘峰，戴洁，程旭，有机化学, 2021, 41, 4014.)

(p) Jiang, J.; Wang, Z.; He, W.-M. Chin. Chem. Lett. 2021, 32, 1591.

(q) Li, M.; Wang, R.; Hao, W.; Jiang, B. Chin. J. Org. Chem. 2020, 40, 1540 (in Chinese).

(李梦帆, 王榕, 郝文娟, 姜波, 有机化学, 2020, 40, 1540.)

(r) Chen, J.-Y.; Lin, Y.-W.; He, W.-M. Chin. Chem. Lett. 2020, 31, 2989.

(s) Gao, J.; Weng, X.; Ma, C.; Xu, X.; Fang, P.; Mei, T. Chin. J. Org. Chem. 2021, 41, 3223 (in Chinese).

(高君青, 翁信军, 马聪, 徐学涛, 方萍, 梅天胜, 有机化学, 2021, 41, 3223.)

(t) Meng, W.; Xu, K.; Guo, B.; Zeng, C. Chin. J. Org. Chem. 2021, 41, 2621 (in Chinese).

(孟薇, 徐坤, 郭兵兵, 曾程初, 有机化学, 2021, 41, 2621.)

(u) Meng, Z.; Feng, C.; Xu, K. Chin. J. Org. Chem. 2021, 41, 2535 (in Chinese).

(蒙泽银, 冯承涛, 徐坤, 有机化学, 2021, 41, 2535.)

(v) Zhou, Y.; Zhao, Z.; Zeng, L.; Li, M.; He, Y.; Gu, L. Chin. J. Org. Chem. 2021, 41, 1072 (in Chinese).

(周娅琴, 赵志恒, 曾亮, 李鸣, 何永辉, 谷利军, 有机化学, 2021, 41, 1072.)

(w) Wang, N.; Xu, J.; Mei, H.; Moriwaki, H.; Izawa, K.; Soloshonol, V.; Han, J. Chin. J. Org. Chem. 2021, 41, 3034 (in Chinese).

(王娜娜，徐敬成，梅海波, Hiroki Moriwaki, Kunisuke Izawa, Va$\operatorname{dim}$ A. Soloshonok, 韩建林, 有机化学, 2021, 41, 3034.)

[5] (a) Wang, B.; Liu, Z.; Tong, Z.; Gao, B.; Ding, H. Angew. Chem. Int. Ed. 2021, 60, 148926.

(b) Wu, Y; Chen, J.-Y.; Ning, J.; Jiang, X.; Deng, J.; Deng, Y.; Xu, R.; He, W.-M. Green Chem. 2021, 23, 3950.

(c) Kai, S.; Xiao, F.; Yu, B.; He, W.-M. Chin. J. Catal. 2021, 42, 1921.

(d) Chen, J.-Y.; Wu, H.-Y.; Gui, Q.-W.; Yan, S.-S.; Deng, J.; Lin, Y.-W.; Cao, Z. He, W.-M. Chin. J. Catal. 2021, 42, 1445.

(e) Wu, Z.-L.; Chen, J.-Y.; Tian, X.-Z.; Ouyang, W.-T.; Zhang, Z.-T.; He, W.-M. Chin. Chem. Lett. 2021, DOI: 10.1016/j.cclet. 2021.08.071.

(f) Chen, J.-Y.; Zhong, C.-T.; Gui, Q.-W.; Zhou, Y.-M.; Fang, Y.-Y.; Liu, K.-J.; Lin, Y.-W.; Cao, Z.; He, W.-M. Chin. Chem. Lett. 2021, 32,475 .

(g) Yi, R.-N.; Wu, Z.-L.; Ouyang, W.-T.; Wang, W.-F.; He, W.-M. Tetrahedron Lett. 2021, 77, 153257.

(h) Chen, J.-Y.; Wu, H.-Y.; Gui, Q.-W.; Han, X.-R.; Wu, Y.; Du, K.; Cao, Z.; Lin, Y.-W.; He, W.-M. Org. Lett. 2020, 22, 2206.

(i) Xiong, Y.; Zhang, J.; Shen, Q.; Huang, J.; Wang, T. Chin. J. Org. Chem. 2021, 41, 2735 (in Chinese)

(熊云奎, 张健叶, 申裙, 黄嘉宇, 王涛, 有机化学, 2021，41, 2735.)

(j) Ma, H.; Mei, T. Chin. J. Org. Chem. 2020, 40, 3982 (in Chinese).

(马红星, 梅天胜, 有机化学, 2020, 40, 3982.)

(k) Jiang, Y.-Y.; Zeng, C.-C. Chin. J. Org. Chem. 2020, 40, 2999 (in 
Chinese).

(蒋洋叶, 曾程初, 有机化学, 2020, 40, 2999.)

(1) Ye, Z.; Zhang, F. Chin. J. Org. Chem. 2020, 40, 241 (in Chinese).

(叶增辉, 张逢质, 有机化学, 2020, 40, 241.)

(m) Feng, E.; Hou, Z.; Xu, H. Chin. J. Org. Chem. 2019, 39, 1424 (in Chinese).

(冯恩祺，侯中伟，徐海超，有机化学, 2019, 39, 1424.)

[6] Yuan, Y.; Lei, A. Nat. Commun. 2020, 11, 802.

[7] Liu, K.; Song, C.; Lei, A. Org. Biomol. Chem. 2018, 16, 2375.

[8] Zhang, J.; Shi, S.-Q.; Hao, W.-J.; Dong, G.-Y.; Tu, S.-J.; Jiang, B. J. Org. Chem. 2021, 86, 15886.

[9] Meng, X.; Xu, H.; Cao, X.; Cai, X.; Luo, J.; Wang, F.; Huang, S. Org. Lett. 2020, 22, 6827.

[10] Li, J.-H.; Qin, J.-H.; Luo, M.-J.; An, D.-L. Angew. Chem. Int. Ed. 2020, 60, 1861.

[11] (a) Wang, F.; Stahl, S. S. Angew. Chem. Int. Ed. 2019, 58, 6385.

(b) Mo, Z.-Y.; Swaroop, T. R.; Tong, W.; Zhang, Y.-Z.; Tang, H.-T.;
Pan, Y.-M.; Sun, H.-B.; Chen, Z.-F. Green Chem. 2018, 20, 4428.

(c) Scheide, M. R.; Nicoleti, C. R.; Martins, G. M.; Braga, A. L. Org. Biomol. Chem. 2021, 19, 2578.

[12] (a) Zhang, T.-S.; Hao, W.-J.; Wang, R.; Wang, S.-C.; Tu, S.-J.; Jiang, B. Green Chem. 2020, 22, 4259.

(b) Zuo, H.-D.; Hao, W.-J.; Zhu, C.-F.; Guo, C.; Tu, S.-J.; Jiang, B. Org. Lett. 2020, 22, 4471.

[13] (a) Aggarwal, T.; Kumar, S.; Verma, A. K. Org. Biomol. Chem. 2016, 14, 7639.

(b) Liang, S.; Zeng, C.-C.; Luo, X.-G.; Ren, F.-Z.; Tian, H.-Y.; Sun, B.-G.; Little, R. D. Green Chem. 2016, 18, 2222.

(c) Midorikawa, K.; Suga, S.; Yoshida, J. Chem. Commun. 2006, 3794.

[14] Yin, Y.; Ma, W.; Chai, Z.; Zhao, G. J. Org. Chem. 2007, 72, 5731.

[15] (a) Voth, C. N.; Dake, G. R. Eur. J. Org. Chem. 2020, 2020, 744.

(b) Abe, M.; Jean, A.; Blanchet, J.; Rouden, J.; Maddaluno, J.; Paolis, M. D. J. Org. Chem. 2019, 84, 15448. 